\title{
Liquid Crystal Temperature Sensor Based on a Micrometric Structure and a Metallic Nanometric Layer
}

\author{
José Francisco Algorri, Virginia Urruchi, Noureddine Bennis,
} and José Manuel Sánchez-Pena

\begin{abstract}
This letter presents a novel temperature sensor, which consists of an interdigitated comb electrode structure with a micrometric-scale size, nanometric metallic layer, and nematic liquid crystal (NLC) film. This sensor exploits the permittivity dependence of the NLC with temperature and principle of electrical conductivity above the percolation threshold in thin film metallic layers. The latter has been demonstrated to increase the temperature sensitivity considerably. The high impedance input reduces the power dissipation, and the high enough voltage output makes it easy to measure the output signal with high precision. The operation principle and fabrication process as well as the characterization of the temperature sensor are presented. Experimental results show that the device offers a sensitivity of $9 \mathrm{mV} /{ }^{\circ} \mathrm{C}$ and is dependent on the applied voltage. This is six times greater than the same structure without the use of a nanometric layer.
\end{abstract}

Index Terms-Temperature sensors, thin film sensors, liquid crystals, microstructure.

\section{INTRODUCTION}

$\mathbf{T}$ HE well-known electrically-controlled anisotropic properties of liquid crystals not only have been valued in displays applications, but also they have led to the conception of innovative approaches for a wide and diversified field of applications. Furthermore, the fact that NLC parameters have a strong dependence on temperature has allowed researchers to extend the design of NLC devices into the field of temperature sensors. Most of these sensors are based on optical properties of NLC. For example, Hak-Rin Kim et al. introduce a NLC film in Fabry-Perot cavity to obtain lambda shifts of $1.05 \mathrm{~nm} /{ }^{\circ} \mathrm{C}$ for maximum temperatures of $65{ }^{\circ} \mathrm{C}$ [1]. Others works have demonstrated the use of photonic crystal fibers as temperature sensor. For instance, Y. Wang et al. achieved a very high temperature sensitivity, $54.3 \mathrm{~nm} /{ }^{\circ} \mathrm{C}$ but for very small temperature ranges $\left(34{ }^{\circ} \mathrm{C}\right.$ to $\left.35.5{ }^{\circ} \mathrm{C}\right)$ [2]. Also considering this technology, D. J. $\mathrm{Hu}$ et al. produced sensitivities of $-3.9 \mathrm{~nm} /{ }^{\circ} \mathrm{C}$ ranging from $44{ }^{\circ} \mathrm{C}$ to $53{ }^{\circ} \mathrm{C}$ [3]. In addition, another type of technology, which consists of Chiral nematic polymer network, has been used in optical temperature sensors as well [4]. This technology does not require complex conditioning circuitry because the temperature can be measured by observing the change on the pattern of color. In summary, these systems have low temperature ranges and sensitivities. Most of them are complex to build, or take measurements (a spectrum analyzer is frequently required). Moving to lower frequencies (electrical signals), permittivity is also very dependent on temperature. Despite this, very few works can be found in the literature that exploits this fact. For example, C. Marcos et al, reported a novel frequencytemperature liquid crystal transducer based on the permittivity change with temperature [5]. A broad temperature range is demonstrated $\left(-6{ }^{\circ} \mathrm{C}\right.$ to $\left.110^{\circ} \mathrm{C}\right)$. In spite of this, the sensitivity is directly proportional to the permittivity change with temperature (by the multivibrator oscillator circuit equation). Special equipment to measure the output or signal conditioning is required.

In this letter, we propose the implementation of a novel kind of NLC temperature sensor, based on an Indium Tin Oxide (ITO) interdigitated comb electrode structure with a micrometer-scale size, and a NLC film. Thanks to this structure, the permittivity change with temperature is introduced in a hyperbolic cosine function that increases the sensitivity term. Additionally, the conformation of the sensor includes a metallic layer (Nickel) of nanometric thickness deposited onto the ITO electrodes. The benefit derived from using this layer is the inclusion of a new factor in the sensitivity term, increasing it considerably.

\section{Operational PRinciple}

The structure and dimensions of the LC device are determinant in order to have a high enough voltage output. Two interlaced combs are designed: The first one, Comb 1, acts as voltage source. Comb 2 measures the voltage drop at the central point between two teeth of the Comb 1. The device conductivity is increased by depositing Nickel (Ni) that forms aggregated Nanoparticles (NP) onto a $\mathrm{SiO}_{2}$ layer by 


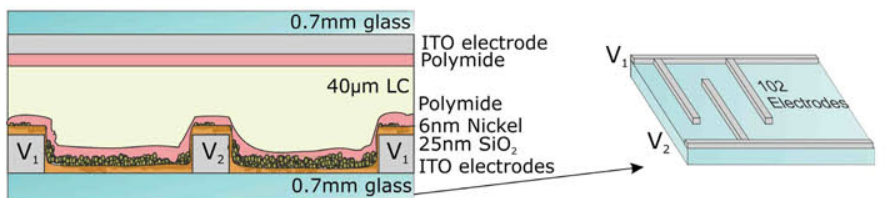

a)

b)

Fig. 1. Sensor diagram. (a) Cross sectional view of the proposed sensor, (b) detail of the comb electrodes. Note that sizes are not to scale.

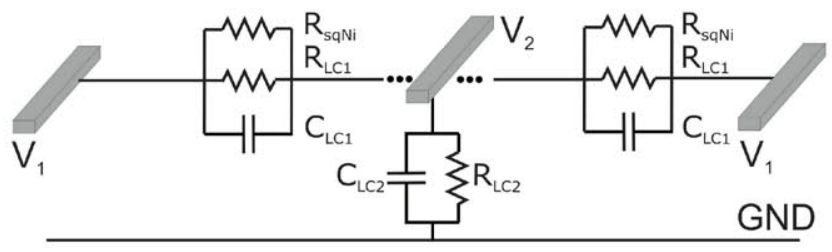

Fig. 2. Simplified EEC based on transmission line theory.

an evaporation process. The governing equations of the proposed structure can be derived from the equivalent electrical circuit (EEC) (see Fig. 2).

The gradual voltage between electrodes has been modeled by considering the sensor as a transmission line [6].

$$
\frac{\partial V^{2}(x)}{\partial x^{2}}=\frac{1 / R_{L C 2}+j \omega C_{L C 2}}{1 / R_{S q N i}+j \omega C_{L C 1}} \cdot V(x)=\Psi \cdot V(x)
$$

The conductivity of the $\mathrm{Ni}$ layer is modeled as a resistor $\left(R_{s q N i}\right)$. The other components can be simplified. The parallel resistor $\left(R_{L C 1}\right)$ and capacitor $\left(C_{L C 1}\right)$ of the NLC are higher than $R_{s q N i}$ so they are negligible. This fact is possibly the main reason of the sensitivity increase; it is explained in section IV. In the range of $100 \mathrm{~Hz}-10 \mathrm{kHz}$ the impedance produced by $C_{L C 2}$ is smaller than $R_{L C 2}$, so this term can be depreciated. The relation between $C_{L C 2}$ and the structural dimensions of the sensor is,

$$
C_{L C 2}=\varepsilon_{0} \cdot \varepsilon_{L C 2}^{\prime} / t_{L C}\left(F / m^{2}\right)
$$

where $\varepsilon_{0}$ is the vacuum permittivity, $t_{L C}$ the LC thickness and $\varepsilon_{L C 2}^{\prime}$ the average real effective permittivity in the vertical direction. If only one harmonic is considered and $\mathrm{V}(\mathrm{x}=-\mathrm{r})=$ $\mathrm{V}(\mathrm{x}=\mathrm{r})=V_{1}$, and $\mathrm{V}(\mathrm{x}=0)=V_{2}(\mathrm{r}$ is the distance between electrodes), the solution of (1) is,

$$
V_{2}=V_{1} / \cosh \left(\sqrt{\frac{R_{s q N i} \varepsilon_{0} \varepsilon^{\prime} L C 2 \omega}{2 t_{L C}}} \cdot r\right)
$$

From Eq. 3 we can predict the offset value for the sensor; this value is directly proportional to $V_{1}$. The parameter that gives the sensor sensitivity is $\varepsilon_{L C 2}^{\prime}$. As more voltage is applied, the real permittivity parameter changes from a low value (ordinary permittivity, $\varepsilon_{o}^{\prime}$ ) to a higher one (extraordinary permittivity, $\varepsilon_{e}^{\prime}$ ), following a non-linear curve. It is a known fact that extraordinary permittivity is more dependent on temperature changes [7], so as $V_{1}$ is increased the sensitivity should increase too. The inclusion of this parameter in a hyperbolic function increments this effect considerably. Low values of $R_{s q N i}$ could lead to a hyperbolic cosine equal to one. In this case, the result is $V_{2}=V_{1}$ and the output would not change with temperature.

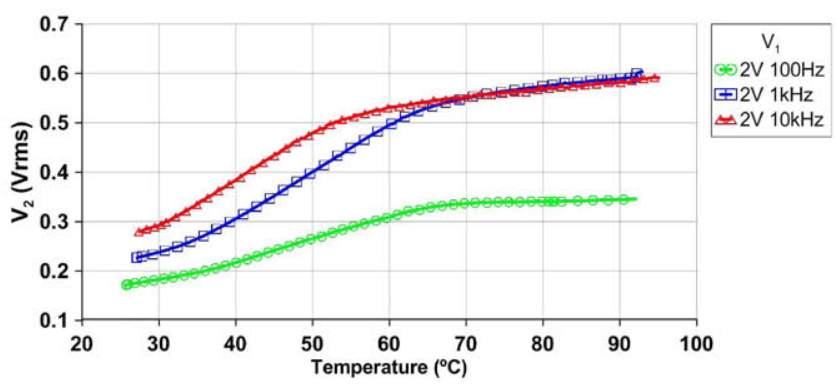

Fig. 3. Frequency dependence of LC sensor response.

\section{FABRICATION AND MEASUREMENT}

Two ITO-Coatings on high quality glass are used as substrates. A chrome mask specially designed to use in high resolution lithography is needed to make the comb electrodes. On one glass substrate [Fig. 1(b)] the electrodes width is $20 \mu \mathrm{m}$ and the distance between electrodes is $107 \mu \mathrm{m}$. There are two combs of electrodes composed of 51 electrodes each. The active area is scalable by including more or less electrodes. In this case, the size is $1.78 \mathrm{~cm}^{2}$. A $25 \mathrm{~nm} \mathrm{SiO} 2$ layer is evaporated in a vacuum chamber. Then $6 \mathrm{~nm}$ of $\mathrm{Ni}$ are evaporated onto this layer. Finally, a polyimide layer is deposited and rubbed in the electrode direction (homogeneous alignment). The other substrate has a continuous conducting layer of ITO. Spacers of $40 \mu \mathrm{m}$ are used to separate both substrates. The resulting cavity is filled with a commercial NLC MDA-98-1602 from Merck. The dielectric anisotropy is $\Delta \varepsilon=12$; the real extraordinary and ordinary permittivities at $1 \mathrm{kHz}$ are, $\varepsilon_{\mathrm{e}}^{\prime}=16.2$ and $\varepsilon_{\mathrm{o}}^{\prime}=4.3$, respectively. The imaginary part of the permittivity $\left(\varepsilon^{\prime \prime}\right)$ is not provided. The clearing point is $109{ }^{\circ} \mathrm{C}$ and the minimum operating temperature is $-20{ }^{\circ} \mathrm{C}$. The typical behavior of the permittivity as a function of temperature can be found in ref. [7].

The electrical characterization of the device was carried out by using an automated system based on MATLAB ${ }^{\circledR}$ and a microcontroller. The temperature is increased at a rate of $1{ }^{\circ} \mathrm{C} / \mathrm{min}$. The supply voltage $\left(V_{1}\right)$ is controlled by a waveform generator. Square signals with variable frequency and amplitude have been employed. The sensor is inside of a LTS350 heating and freezing stage. In order to control the temperature in both sides of the substrates, two silicon sensors (LM35) are used to take temperature measures. The voltage output $\left(V_{2}\right)$ is connected to an operational amplifier based buffer. The output of this buffer is converted to a dc signal by an AD536 RMS/DC converter. This output and LM35 sensors are connected to an ATMEGA 2560 microcontroller.

\section{RESUlTS AND Discussion}

The first experiment consists of variations of the applied voltage frequency with a fixed amplitude (Fig. 3). All the measurements have been done in an increasing and decreasing direction and no hysteresis has been observed. The resolution is limited by the LM35 sensors $\left(0.5{ }^{\circ} \mathrm{C}\right)$. For $1 \mathrm{kHz}$ the sensitivity is highest. At this frequency the real permittivity $\left(\varepsilon_{L C 2}^{\prime}\right)$ is predominant, so one reason for this behavior could 


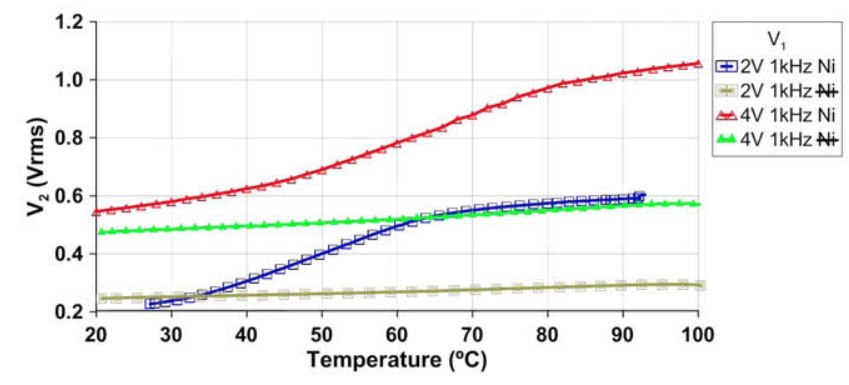

Fig. 4. Voltage (rms) dependence of LC sensor response for cells fabricated with Ni layer and without it.

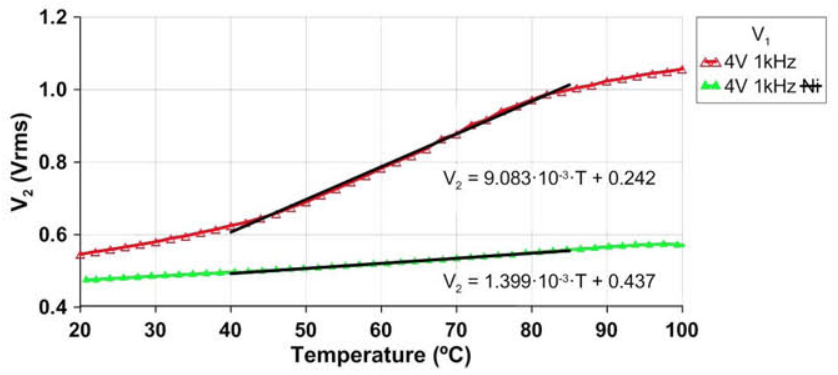

Fig. 5. Linear fittings to sensor responses.

be greater changes of the real permittivity with temperature than the imaginary permittivity $\left(\varepsilon_{L C 2}^{\prime \prime}\right)$. In order to demonstrate the Ni nanometric layer effect, another device with the same dimensions but without this layer is fabricated and characterized (Fig. 4).

For the case of the sensor without $\mathrm{Ni}$, high voltages have been tested. Maximum sensitivities of $33 \mathrm{mV} /{ }^{\circ} \mathrm{C}$, for certain temperature ranges $\left(60^{\circ} \mathrm{C}-95^{\circ} \mathrm{C}\right)$, have been obtained. Further experiments have to be made in order to study the operating limit of the Ni sensor. The maximum current allowed by this layer will be a key parameter in order to apply more voltage. Higher sensitivities than the sensor without $\mathrm{Ni}$ are expected.

The applied voltage $\left(V_{1}\right)$ affects the sensitivity because the extraordinary component is more temperature dependent, $d \varepsilon_{e}^{\prime}(\mathrm{T}) / d \mathrm{~T}$ greater than $d \varepsilon_{o}^{\prime}(\mathrm{T}) / d \mathrm{~T}$ [7]. This fact indicates that a homogeneous alignment, where all the molecules are perpendicular without any applied voltage, could lead to higher sensitivities for very low voltages. As can be observed in Fig. 4, the use of a Ni nanometric layer resulted in significant differences in the sensitivity.

In Fig. 5 the difference between these two types of sensors and a linear fit is shown. The temperature sensitivity of the device with the Ni nanometric layer is almost six times greater, demonstrating a significant increase of this one with regard to the other structure. Possibly the introduction of $R_{s q N i}$ parameter in (1) (resulting in the suppression of $C_{L C 1}$ ) makes the sensitivity increase. As $C_{L C 1}$ is also temperature dependent and is dividing $C_{L C 2}$, if it had been considered, it would have reduced the sensitivity considerably.

As commented above, the application of higher voltages to the $\mathrm{Ni}$ layer has to be further investigated. These results indicate a clear tendency to higher sensitivities. Despite this, considering the presented sensitivity, this sensor has clear advantages over other proposed sensors. The main one is an easy integration in Liquid Crystal Displays (LCDs) due to the similarity of these technologies. The structure is simple and has an active area easy to scale. Coplanar electrodes can be easily patterned at very small dimensions yielding miniaturized, reproducible, and ultimately low cost devices.

\section{CONCLUSION}

The LC sensor shows that the output voltage's sensitivity to temperature response can be controlled by either the magnitude or the frequency of the applied voltage. For certain supply voltages, this sensor has demonstrated high temperature sensitivity. In addition to low power consumption, improves sensor features in relation to other reported temperature sensors. This sensor could be used in LCDs, LCD projectors, LCDs that are used in extreme environments (outdoor, avionics display, military aerospace display), portable equipment or any application where its properties get an advantage with respect to current available sensors. It also could be an interesting application for autostereoscopic devices based on LC. A homeotropic alignment would make better use of the temperature permittivity dependence because the maximum sensitivity would be for low voltages. The inclusion of a $\mathrm{Ni}$ nanometric layer improves the sensitivity of the sensor by six times. This work has presented and characterized a novel idea, and opened new avenues for research on temperature sensors.

\section{REFERENCES}

[1] H. R. Kim, E. Jang, and S. D. Lee, "Electrooptic temperature sensor based on a Fabry-Pérot resonator with a liquid crystal film," IEEE Photon. Technol. Lett., vol. 18, no. 8, pp. 905-907, Apr. 15, 2006.

[2] Y. Wang et al., "Selectively infiltrated photonic crystal fiber with ultrahigh temperature sensitivity," IEEE Photon. Technol. Lett., vol. 23, no. 20 , pp. 1520-1522, Oct. 15, 2011.

[3] D. J. J. Hu et al., "Fabrication and characterization of a highly temperature sensitive device based on nematic liquid crystal-filled photonic crystal fiber," IEEE Photon. J., vol. 4, no. 5, pp. 1248-1255, Oct. 2012.

[4] D. J. D. Davies et al., "A printable optical time-temperature integrator based on shape memory in a chiral nematic polymer network," $A d v$. Funct. Mater, vol. 23, no. 21, pp. 2665-2670, Jun. 2013.

[5] C. Marcos et al., "Temperature-frequency converter using a liquid crystal cell as a sensing element," Sensors, vol. 12, no. 3, pp. 3204-3214, Mar. 2012.

[6] V. Urruchi et al., "Electrical modeling and characterization of voltage gradient in liquid crystal microlenses," Rev. Sci. Instrum., vol. 84, no. 11, p. 116105, Nov. 2013.

[7] L. M. Blinov, "Magnetic, electric and transport properties," in Structure and Properties of Liquid Crystals. New York, NY, USA: Springer-Verlag, 2011, p. 162. 